\title{
Plate Element Formulation for a Discrete Element Method
}

\author{
Dr. C. Mariotti \\ CEA, DAM, DIF, F.91297 Arpajon, France \\ christian.mariotti@cea.fr
}

\begin{abstract}
The discrete elements code CeaMka3D uses cells of Voronoï as particles. Having clarified the expression of forces and torques in the elastic formulation, the concepts of volume of rotation and of volumetric vector of bending are introduced to simulate the bending. Some simulations of plates illustrate the capacity of this formulation.
\end{abstract}

Keywords: Discrete element method; elasticity; thin plate; bending; CeaMka3D

\section{Introduction}

Particle methods are meshless simulation techniques in which a continuum medium is approximated through the dynamics of a set of interacting solids. These include the Discrete Element methods (DEM) first developed by Hoover, Arhurst and Olness [1] in models for crystalline materials. They were applied to geotechnical problems by Cundall and Strack [2], and they are still in widespread use for granular materials and rock simulation [3].

A Discrete Element code CeaMka3D has been developed [4-5]. This method has been used successfully to simulate, for example, the propagation of seismic waves in a linear elastic medium [6]. This code has also been coupled with a finite element code [7] and a new symplectic leapfrog scheme has been developed in order to integrate 3D rigid-body rotation with external torque [8].

In the first part, the forces and torques between two particles are described in order to recover Hooke's law. In the second part, bending moments between two particles are presented. In the last part, the results of this method on a conventional test are presented.

\section{Normal Force between Particles}

The initial choice was to take a Voronoï mesh which allows, from a field of points, to bound polyhedrons. This type of meshing does not leave geometrical space like that can be the case with spherical particles. By geometrical construction, the plan of contact between two particles is perpendicular to the line connecting the centres of particles. 


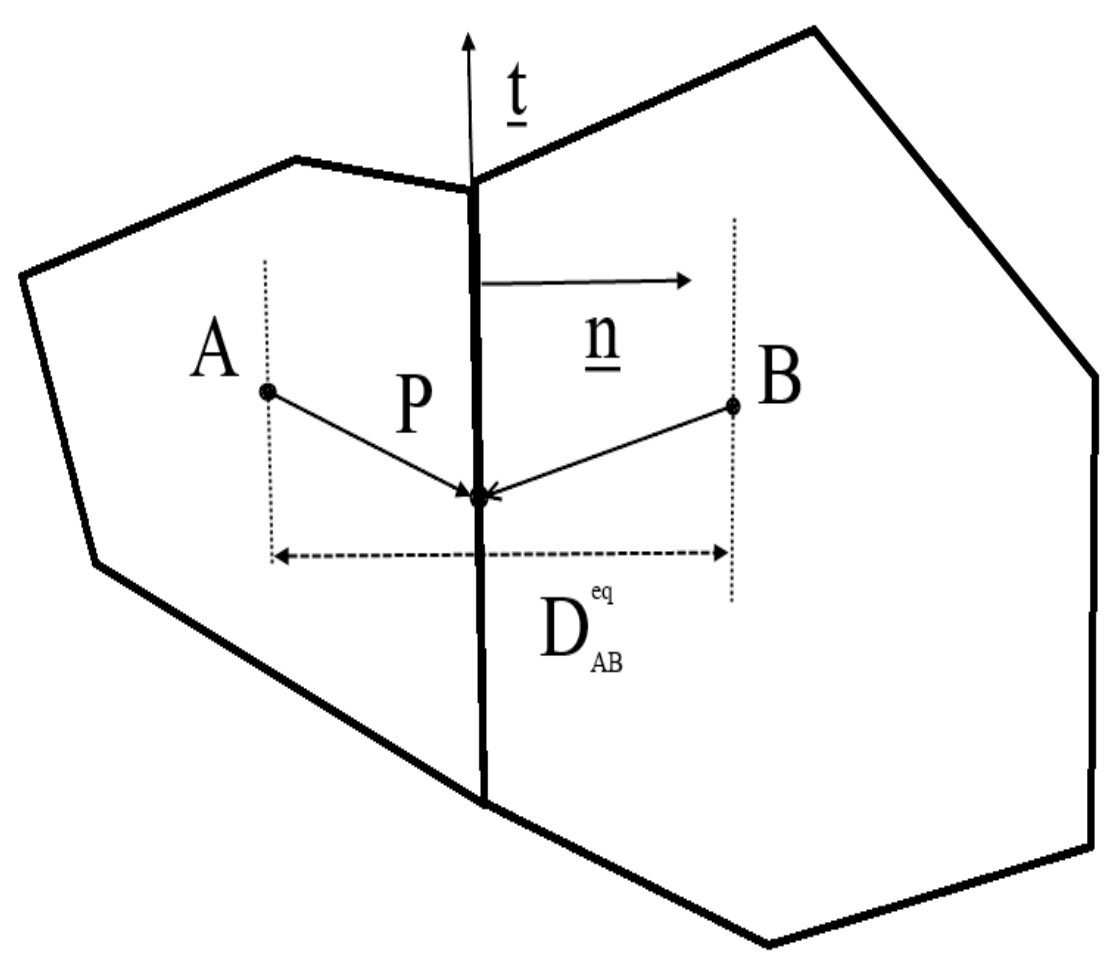

Figure 1. Initial Contact between Two Particles

Let $\underline{n}$ be the normal direction between particles $A$ and $B$

$$
\underline{n}=\frac{\underline{A B}}{\|\underline{A B}\|} .
$$

The initial distance between particles $A$ and $B$ is defined by

$$
D_{A B}^{e q}=\|\underline{A B}\|_{t=0} .
$$

The relative movement of both particles $A$ and $B$ according to the normal is defined by

$$
D_{A B}^{n}=D_{A B}^{e q}-\|\underline{A B}\| .
$$

It is necessary to choose the method of calculation of the elastic volume deformation of a particle $A$ surrounded by other particles $B$ during their movements.

A particle $A$ has only a part of its surface in touch with the other particles B. A volume of contact can be defined by the following relation

$$
V_{A}^{C}=\frac{1}{3} \sum_{\text {links }} \frac{1}{B} 2 D_{A B}^{e q} S_{A B}
$$

where $S_{A B}$ is the contact area between particles $A$ and $B$.

The variation of this volume of contact is given by the following equation

$$
\Delta V_{A}^{C}=\sum_{\text {links }} \frac{1}{B} \frac{1}{2} S_{A B} D_{A B}^{n} .
$$


To transform this variation of elastic volume of contact to the elastic volume deformation, it is necessary to integrate free surfaces of the particle which can also have an elastic deformation.

The complementary volume, called free volume, is then defined by

$$
V_{A}^{F}=V_{A}-V_{A}^{C} .
$$

The variation of elastic volume of the particle $A$ is given by the relation

$$
\varepsilon_{A}^{v}=\varepsilon_{A}^{v C}+\varepsilon_{A}^{v F}=\frac{\Delta V_{A}^{C}+\Delta V_{A}^{F}}{V_{A}} .
$$

To define the variation of free volume, it is necessary to return to the Hooke's law. On the free surface and according to the normal for this free surface, the normal constraint is nil, so

$$
\varepsilon_{A}^{n n}=\frac{-v}{1-2 v} \varepsilon_{A}^{v},
$$

where $v$ is Poisson's ratio.

On the other hand, the normal elastic deformation of the free surface is connected with the variation of the free volume

$$
\varepsilon_{A}^{n n}=\frac{1}{3} \frac{\Delta V_{A}^{F}}{V_{A}^{F}}
$$

So

$$
\varepsilon_{A}^{v F}=\frac{\Delta V_{A}^{F}}{V_{A}}=-3 \frac{v}{1-2 v}\left(\frac{V_{A}^{F}}{V_{A}}\right) \varepsilon_{A}^{v}=-3 \frac{v}{1-2 v}\left(1-\frac{V_{A}^{C}}{V_{A}}\right) \varepsilon_{A}^{v},
$$

and

$$
\varepsilon_{A}^{v C}=\frac{\Delta V_{A}^{C}}{V_{A}}
$$

Finally

$$
\varepsilon_{A}^{v}=\varepsilon_{A}^{v C}+\varepsilon_{A}^{v F}=\frac{\Delta V_{A}^{C}}{V_{A}}-3 \frac{v}{1-2 v}\left(1-\frac{V_{A}^{C}}{V_{A}}\right) \varepsilon_{A}^{v}=\frac{\Delta V_{A}^{C}}{V_{A}}\left|\frac{1}{1+3 \frac{v}{1-2 v}\left(1-\frac{V_{A}^{C}}{V_{A}}\right)}\right|
$$

So, the elastic volume deformation of a particle $\mathrm{A}$ is given by

$$
\varepsilon_{A}^{v}=\frac{1}{V_{A}}\left|\frac{1}{1+3 \frac{v}{1-2 v}\left(1-\frac{V_{A}^{C}}{V_{A}}\right)}\right| \sum_{\mid \text {links }} \frac{1}{2} S_{B} S_{A B} D_{A B}^{n} .
$$

In the expression of the normal force between particles $A$ and $B$, the following volume deformation is defined by 


$$
\varepsilon_{A B}^{v}=\frac{1}{2}\left(\varepsilon_{A}^{v}+\varepsilon_{B}^{v}\right) .
$$

The normal force between both particles $A$ and $B$ is then given by

$$
\underline{F}_{A B}^{n}=\left(K_{s} \frac{D_{A B}^{n}}{D_{A B}^{e q}}+K_{v} \varepsilon_{A B}^{v}\right) S_{A B} \underline{n}
$$

with

$$
\begin{aligned}
& K_{s}=\frac{E}{1+v} \\
& K_{v}=\frac{E v}{(1+v)(1-2 v)} .
\end{aligned}
$$

with E Young's modulus and v Poisson's ratio.

The free surface is also important for the bending and a volume of rotation is defined by

$$
V_{A}^{\theta}=V_{A}\left(1+3 \frac{v}{1-2 v}\left(1-\frac{V_{A}^{C}}{V_{A}}\right)\right) .
$$

The expressions of the shear forces and torques associated are studied in the next section.

\section{Shear Forces and Torques Associated}

The variation of the shear displacement is defined by the following vector

$$
\underline{\Delta u}_{A B}^{t}=\left(\underline{A B}-\underline{\underline{r o t}} A \underline{A P_{A B}^{0}}+\underline{\underline{r o t}} B B P_{A B}^{0}\right)-\left(\underline{A B}-\underline{\underline{r o t}} A \underline{A P}_{A B}^{0}+\underline{\underline{r o t}}{ }_{B} B P_{A B}^{0}\right) \bullet \underline{n} \underline{n}
$$

where - indicates the scalar product and $\underline{\underline{r o t}}{ }_{A}$ is the matrix of rotation of the particle A.

The shear force between particles is given by

$$
\underline{F}_{A B}^{t}=-K_{s} \frac{S_{A B}}{D_{A B}^{e q}} \underline{\Delta u}_{A B}^{t} .
$$

The torques are given by

$$
\begin{aligned}
& \underline{M}_{A}^{t}=-\underline{\underline{r o t}}{ }_{A} \underline{A P}_{A B}^{0} \wedge \underline{F}_{A B}^{t}, \\
& \underline{M}_{B}^{t}=\underline{\underline{r o t}} B{ }_{B} B P_{A B}^{0} \wedge \underline{F}_{A B}^{t},
\end{aligned}
$$

where $\wedge$ is the cross product.

The terms of bending and torsion are examined in the next section.

\section{Bending and Torsion}

For a link between two particles $A$ and $B$, a frame is associated with the initial contact surface $S_{A B}$ between $A$ and $B$. This frame consists of three vectors $(\underline{n}, \underline{s}, \underline{t})$. The inertia 
tensor of the contact surface is calculated with regard to both principal directions $\underline{s}$ and $\underline{t}$ from the centre of gravity $G$ of this surface

$$
\begin{aligned}
& I_{A B}^{s}=\iint_{\text {sufface }}(\underline{G P} \bullet \underline{s})^{2} d S, \\
& I_{A B}^{t}=\iint_{\text {sufface }}(\underline{G P} \bullet \underline{t})^{2} d S .
\end{aligned}
$$

Two reduced distances according to each of these directions are defined by

$$
\begin{aligned}
& d_{A B}^{s}=\sqrt{\frac{I_{A B}^{s}}{S_{A B}}}, \\
& d_{A B}^{t}=\sqrt{\frac{I_{A B}^{t}}{S_{A B}}} .
\end{aligned}
$$

The normal direction $\underline{n}$ will be associated with a torsional torque $J$.

Each particle $A$ is going to have its own matrix of rotation $\underline{\underline{\underline{r o t}}}$ A and some vectors are defined by

$$
\begin{aligned}
& \underline{n}_{A B}^{A}=\underline{\underline{r o t}}_{A} \underline{n}, \\
& \underline{n}_{A B}^{B}=\underline{\underline{r o t}}{ }_{B} \underline{n}, \\
& \underline{s}_{A B}^{A}=\underline{\underline{r o t}}{ }_{A} \underline{s}, \\
& \underline{s}_{A B}^{B}=\underline{\underline{r o t}}_{B} \underline{s}, \\
& \underline{t}_{A B}^{A}=\underline{\underline{r o t}}{ }_{A}, \\
& \underline{t}_{A B}^{B}=\underline{\underline{\underline{r o t}}}{ }_{B} \underline{t} .
\end{aligned}
$$

For the bending and torsion, the following potential is taken

$$
E^{\text {flex }}=\frac{1}{2} K_{s} \frac{S_{A B}}{D_{A B}^{e q}}\left(\frac{J_{A B}}{S_{A B}} s t \underset{A B}{2}+\frac{I_{A B}^{t}}{S_{A B}} t n_{A B}^{2}+\frac{I_{A B}^{s}}{S_{A B}} n s_{A B}^{2}\right),
$$

with the following variables

$$
\begin{aligned}
& s t_{A B}=\frac{\underline{\underline{s}}_{A B}^{A} \bullet \underline{t}_{A B}^{B}-\underline{s}_{A B}^{B} \bullet \underline{t}_{A B}^{A}}{2}, \\
& t n_{A B}=\frac{\underline{t}_{A B}^{A} \bullet \underline{n}_{A B}^{B}-\underline{t}_{-A B}^{B} \bullet \underline{n}_{A B}^{A}}{2}, \\
& n s_{A B}=\frac{\underline{n}_{A B}^{A} \bullet \underline{s}_{A B}^{B}-\underline{n}_{A B}^{B} \bullet \underline{s}_{A B}^{A}}{2} .
\end{aligned}
$$

So, the bending and torsion for the particle A are 


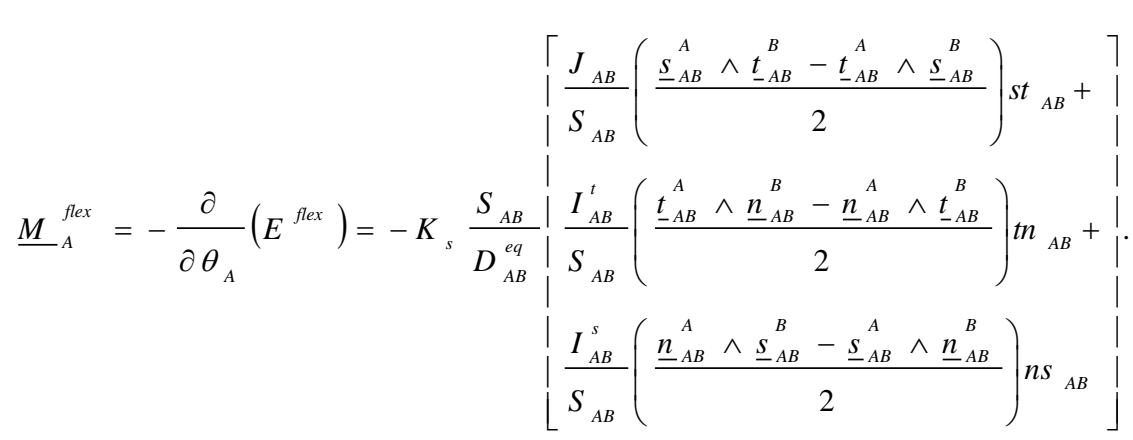

For the normal force, a volume deformation has been defined to simulate correctly the elasticity. In the same way, a vector of volume bending variation can be defined. In the case of a cube submitted to constraints, every face is going to deform. If faces remain flat, the rotation of a face around its centre of gravity does not change the global volume of the cube but there are local volume variations inside the cube. Also, these local volume variations connected to the rotation do not change global forces but on the other hand change the bending when the Poisson's ratio is different from zero.

For the link between particles $A$ and $B$, two vectors defining bending can then be defined

$$
\begin{aligned}
& \underline{V}_{A B}^{s}=\frac{\underline{s}_{A B}^{A} \wedge \underline{s}_{A B}^{B}+\underline{n}_{A B}^{A} \wedge \underline{n}_{A B}^{B}}{2}, \\
& \underline{V}_{A B}^{t}=\frac{\underline{t}_{A B}^{A} \wedge \underline{t}_{A B}^{B}+\underline{n}_{A B}^{A} \wedge \underline{n}_{A B}^{B}}{2} .
\end{aligned}
$$

A volume variation related to bending of link $A B$ is defined for particle $A$ by the vector

$$
\underline{u}_{A B}^{A}=d_{A B}^{s}\left(\underline{V}_{A B}^{s} \bullet \underline{t}_{A B}^{A}\right) \underline{s}_{A B}^{A}-d_{A B}^{t}\left(\underline{V}_{A B}^{t} \bullet \underline{s}_{A B}^{A}\right) \underline{t}_{A B}^{A} .
$$

Similarly, for particle $B$

$$
\underline{u}_{A B}^{B}=d_{A B}^{s}\left(\underline{V}_{A B}^{s} \bullet \underline{t}_{A B}^{B}\right) \underline{s}_{A B}^{B}-d_{A B}^{t}\left(\underline{V}_{A B}^{t} \bullet \underline{s}_{A B}^{B}\right) \underline{t}_{A B}^{B} .
$$

Summing these vector increments for all the links of a particle $A$, an overall bending volume variation vector is defined

$$
\underline{\varepsilon}_{A}^{\theta}=\frac{1}{V_{A}^{\theta}} \sum_{\text {links }} \frac{1}{2} S_{A B} \underline{u}_{A B}^{A} .
$$

The volumetric potential of bending is defined by

$$
E^{\theta}=\frac{1}{2} K_{\mathrm{v}} \sum_{A} V_{A}^{\theta}\left(\varepsilon_{\mathrm{A}}^{\theta}\right)^{2} .
$$

This volumetric potential of bending assures the volume coupling of bending directions.

For a link between a particle $\mathrm{A}$ and a particle $\mathrm{B}$, the torque on $A$ given by this potential is obtained by deriving this potential according to the rotation of the particle $A$ 


$$
\begin{aligned}
& \underline{M}_{A B}^{A \theta}=-K_{\mathrm{v}} V_{A}^{\theta} \underline{\varepsilon}_{A}^{\theta} \frac{\partial}{\partial \theta_{A}}\left(\underline{\varepsilon}_{A}^{\theta}\right)-K_{\mathrm{v}} V_{B}^{\theta} \underline{\varepsilon}_{B}^{\theta} \frac{\partial}{\partial \theta_{A}}\left(\underline{\varepsilon}_{B}^{\theta}\right)=
\end{aligned}
$$

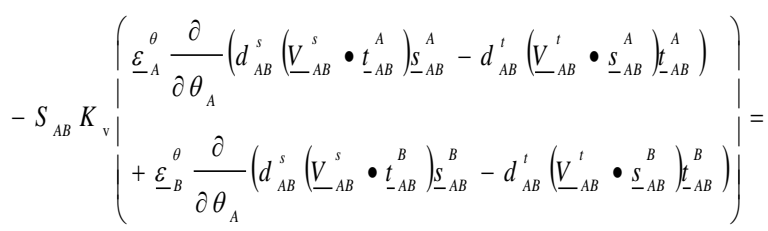

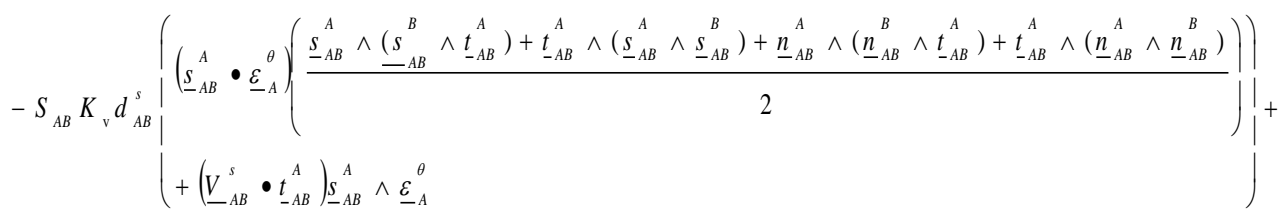

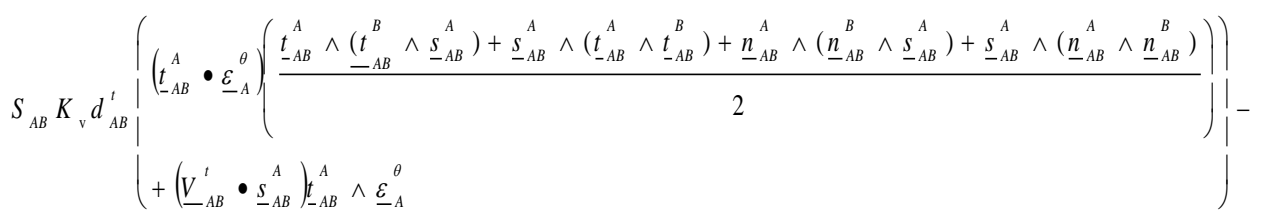

$$
\begin{aligned}
& S_{A B} K_{v} d_{A B}^{s}\left(\underline{s}_{A B}^{B} \bullet \underline{\varepsilon}_{B}^{\theta}\right)\left(\frac{\underline{s}_{A B}^{A} \wedge \underline{n}_{A B}^{B}+\underline{s}_{A B}^{B} \wedge \underline{n}_{A B}^{A}}{2}\right)+S_{A B} K_{v} d_{A B}^{t}\left(t_{-A B}^{B} \bullet \underline{\varepsilon}_{-B}^{\theta}\left(\frac{\underline{n}_{A B}^{B} \wedge \underline{t}_{A B}^{A}+\underline{n}_{A B}^{A} \wedge t_{-A B}^{B}}{2}\right)\right. \text {. }
\end{aligned}
$$

For a plate, the volume of rotation is given by

$$
V^{\theta}=V\left(\frac{1-v}{1-2 v}\right) .
$$

The bending torque takes then the characteristic value of the bending of a plate of thickness $h$

$$
\left(\frac{E}{1+v}+\frac{E v}{(1+v)(1-2 v)} \frac{V}{V^{\theta}}\right) \frac{h^{3}}{12}=\left(\frac{E}{1+v}+\frac{E v}{\left(1-v^{2}\right)}\right) \frac{h^{3}}{12}=\frac{E h^{3}}{12\left(1-v^{2}\right)} .
$$

For a beam, the volume of rotation is given by

$$
V^{\theta}=V\left(\frac{1}{1-2 v}\right) .
$$

The bending torque takes then the characteristic value of the bending of a beam of thickness $h$

$$
\left(\frac{E}{1+v}+\frac{E v}{(1+v)(1-2 v)} \frac{V}{V^{\theta}}\right) \frac{h^{3}}{12}=\left(\frac{E}{1+v}+\frac{E v}{(1+v)}\right) \frac{h^{3}}{12}=E h^{3} .
$$

So, on one hand the volume of rotation assures the consideration of the various geometries (beam, plate ...). On the other hand, the volume vector of bending allows the directional assembly of the bending.

So, these elastic potentials have been defined in order to simulate bending and torsion.

\section{Numerical Verification}

This formulation is verified on a classical benchmark for plates in elasticity.

A circular plate of radius $R=10 \mathrm{~m}$, thickness $h$ and supporting a uniform pressure $P$ is considered. The plate is embedded at the circumference of the circle. 
The deflection is given by the following analytical relation [9]

$$
w(\xi)=\frac{P R^{4}}{64 D}\left(1-\xi^{2}\right)\left(\left(1-\xi^{2}\right)+\varphi\right),
$$

where

$$
\begin{aligned}
& \xi=\frac{r}{R}, \\
& D=\frac{E h^{3}}{12\left(1-v^{2}\right)}, \\
& \varphi=4\left(\frac{h}{R}\right)^{2} \frac{1}{1-v} .
\end{aligned}
$$

At the centre $r=0$, the expected deflection is given by

$$
w(0)=\frac{P R^{4}}{64 D}(1+\varphi) .
$$

A square plate, $22 \mathrm{~m}$ side length, is meshed with just one particle in the thickness but with irregular particles of about $0.5 \mathrm{~m}$ length (mesh with a total of 1936 particles, Figure 2 ). The particles outside the circle of radius $R=10 \mathrm{~m}$ are embedded. This plate is tested for several thicknesses $h=(0.25 \mathrm{~m} ; 0.1 \mathrm{~m} ; 0.025 \mathrm{~m})$ and for several values of Poisson's ratio $v=(0 ; 0.25 ; 0.49)$ and a Young modulus $E=10^{7} \mathrm{~Pa}$. For these nine cases, the value of $P$ is calculated by the analytical relation (44) in order to have always the same central deflection of $10^{-4} \mathrm{~m}$. Simulations are compared with the analytical solution with a relative error less than $0.5 \%$ (Table 1). The amplified deformed shape, with a Poisson's ratio $v=0.49$ and $h=0.25 \mathrm{~m}$, is shown in Figure 2 .

For thin plate $\left(\frac{D_{A B}^{e q}}{h} \geq 2.5\right)$, there is some shear locking and the following reduction of the shear modulus is taken

$$
K_{s}^{*}=K_{s} \frac{a-b}{a+\frac{D_{A B}^{e q}}{h}\left(\operatorname{Ln}\left(\frac{D_{A B}^{e q}}{b h}\right)-1\right)} \text { for } \frac{D_{A B}^{e q}}{h} \geq b
$$

with $\mathrm{a}=2.845$ and $\mathrm{b}=2.5$

Table 1. Central Deflections of the Plate with CeaMka3D

\begin{tabular}{|c|c|c|c|}
\hline $\begin{array}{c}\text { Central deflections } \\
(\mathrm{m})\end{array}$ & $\begin{array}{c}\text { Thickness } \\
h=0.25\end{array}$ & $\begin{array}{c}\text { Thickness } \\
h=0.1\end{array}$ & $\begin{array}{c}\text { Thickness } \\
h=0.025\end{array}$ \\
\hline $\begin{array}{c}\text { Poisson's ratio } \\
v=0\end{array}$ & $0.997610^{-4}$ & $0.997610^{-4}$ & $0.998610^{-4}$ \\
\hline $\begin{array}{c}\text { Poisson's ratio } \\
v=0.25\end{array}$ & $0.998710^{-4}$ & $0.999510^{-4}$ & $1.000210^{-4}$ \\
\hline $\begin{array}{c}\text { Poisson's ratio } \\
v=0.49\end{array}$ & $1.000910^{-4}$ & $1.003010^{-4}$ & $1.004010^{-4}$ \\
\hline
\end{tabular}




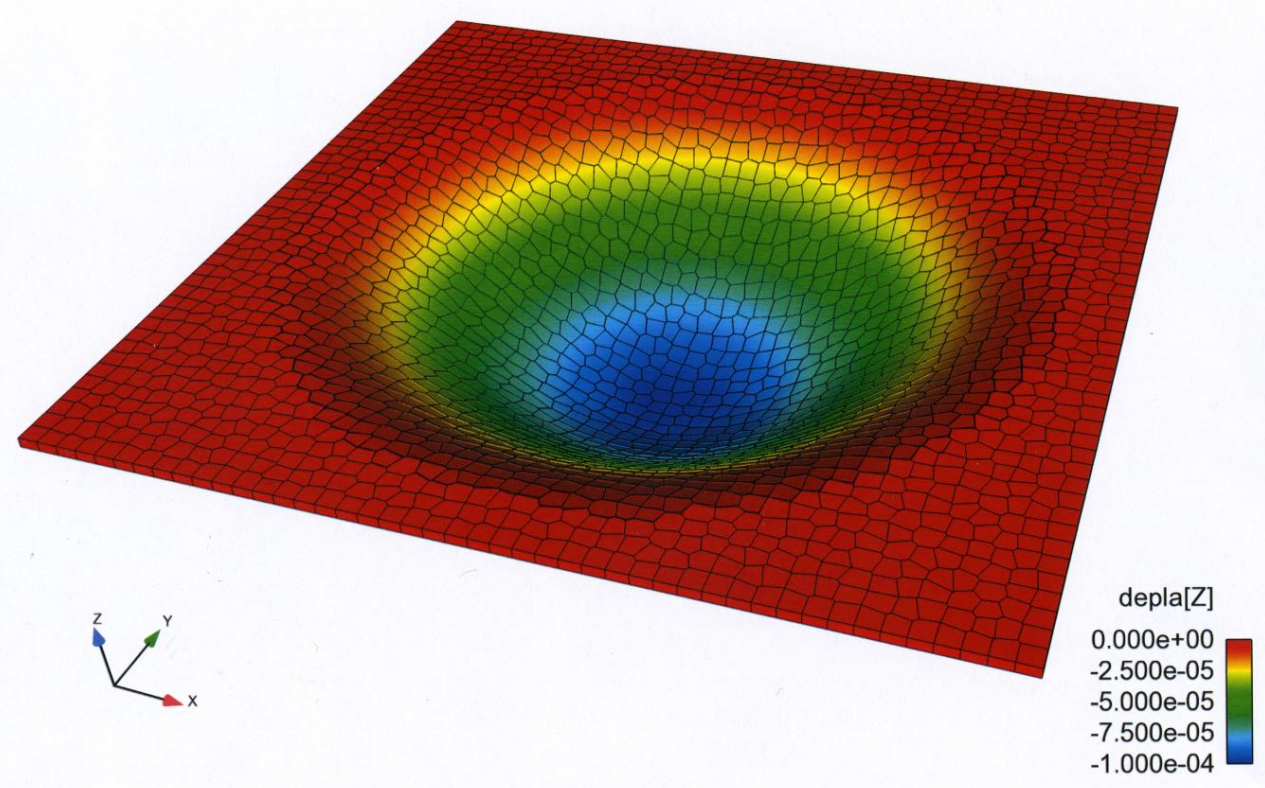

\section{Figure 2. Embedded Circular Plate $(R=10 \mathrm{~m})$ with Irregular Mesh - Poisson's Ratio $v=0.49$ and $\mathrm{h}=0.25 \mathrm{~m}$ - Deformed Shape Amplified 50000 Times}

\section{Conclusion}

The formulation of the discrete elements code CeaMka3D has been presented in the elastic domain. Two important concepts are presented. The first concept is the volume of rotation which allows taking into account various geometries (solid, beam or plate) according to the volume of contact between particles. The second concept is the volumetric vector of bending which allows taking into account the coupling between the bending directions when the Poisson's ratio is different from zero. This formulation is verified on a classical benchmark for plates.

\section{References}

[1] W. G. Hoover, W. T. Arhurst and R. J. Olness, "Two-dimensional studies of crystal stability and fluid viscosity", Journal of Chem Phys, vol. 60, (1974), pp. 4043-4047.

[2] P. A. Cundall and O. D. L. Strack, "A discrete numerical model for granular assemblies", Geotech., vol. 29, no. 1, (1979), pp. 47-65.

[3] D. O. Potyondy and P. A. Cundall, "A bonded-particle model for rock", International Journal of Rock Mech Min Sci., vol. 41, (2004), pp. 1329-1364.

[4] C. Mariotti and L. Monasse, "From general mechanics to discontinuity, unified approach to elasticity", Presses des Ponts, ISBN: 978-2-85978-460-7, (2012).

[5] C. Mariotti, "Lamb's problem with the lattice model Mka3D", Geophys Journal of Int., vol. 171, (2007), pp. 857-864.

[6] L. Monasse and C. Mariotti, "An energy-preserving Discrete Element Method for elastodynamics", ESAIM: M2AN, vol. 46, no. 6, (2012), pp. 1527-1553.

[7] C. Mariotti, F. Le Piver and L. Aubry, "A least-squares coupling method between a finite element code and a discrete element code", Int J Num Meth Eng., vol. 101, no. 10, (2015), pp. 731-743.

[8] C. Mariotti, "A new Leapfrog scheme for rotational motion in 3D", Int J Num Meth Eng doi: 10.1002/nme.5165, (2015)

[9] S. P. Timoshenko and S. Woinowsky-Krieger, "Theory of Plates and Shells", McGraw-Hill, New York, (1959). 
International Journal of Advanced Science and Technology Vol.94 (2016) 